Pacific Journal of Mathematics

ON THE HOMOLOGY OF SPACES OF SECTIONS OF
COMPLEX PROJECTIVE BUNDLES 


\title{
ON THE HOMOLOGY OF SPACES OF SECTIONS OF COMPLEX PROJECTIVE BUNDLES
}

\author{
JESPER Michael MØLler
}

\begin{abstract}
By means of a Moore-Postnikov decomposition we compute the first homology groups of some spaces of sections of projective bundles associated to complex vector bundles.
\end{abstract}

1. Introduction. Let $P \xi: P(V) \rightarrow X$ be the projective bundle associated to a complex $(n+1)$-dimensional vector bundle $\xi: V \rightarrow X, n \geq 1$, over a connected $\mathrm{CW}$-complex $X$. Suppose that $P \xi$ admits a section $u$ : $X \rightarrow P(V)$ and consider the space $\Gamma_{u}$ of all sections vertically homotopic to $u$. In this paper we discuss the (co)-homology of $\Gamma_{u}$ using the construction by Thom-Haefliger [1] of $\Gamma_{u}$ as an inverse limit derived from the Moore-Postnikov factorization of $P \xi$. Explicit formulas for some (co-)homology groups of $\Gamma_{u}$ are obtained provided $X=T$ is a closed, orientable surface, $X=P^{m}, 1 \leq m \leq n$, is a complex projective space, or $X=$ $L^{2 m+1}(p), 1 \leq m<n, p$ odd, is a lens space.

If $\xi$ is trivial, then $\Gamma_{u}$ is a (path-)component of the space $M\left(X, P^{n}\right)$ of maps of $X$ into $P^{n}$, so in particular we obtain formulas for some homology groups of the components of $M\left(X, P^{n}\right)$. In fact, sufficient information is obtained to show that two components of $M\left(T, P^{n}\right)$ or $M\left(P^{m}, P^{n}\right), 1 \leq m \leq n$, are homotopy equivalent if and only if their associated degrees have the same absolute value.

The work presented here was inspired by the paper [4], in turn inspired by [2], in which Larmore and Thomas computed the fundamental group of some spaces of sections of real projective bundles associated to real vector bundles. In contrast to [4] we avoid, however, the use of twisted coefficients, for $P \xi$ is orientable, and the focus will be on homology groups rather than homotopy groups.

2. Moore-Postnikov factorizations of projective bundles. Since the projective bundle $P \xi: P(V) \rightarrow X$, having a connected structure group, is 
orientable, it admits a Moore-Postnikov factorization of the following type

$$
\begin{aligned}
& P(V) \\
& q \downarrow \\
& K(\mathbf{Z} / 2,2 n+2) \quad \rightarrow \quad E_{3} \\
& p_{3} \downarrow \\
& K(\mathbf{Z}, 2 n+1) \quad \rightarrow \quad E_{2} \quad \stackrel{k^{2 n+3}}{\rightarrow} K(\mathbf{Z} / 2,2 n+3) \\
& p_{2} \downarrow \\
& K(\mathbf{Z}, 2) \quad \rightarrow \quad E_{1} \quad \stackrel{k^{2 n+2}}{\rightarrow} \quad K(\mathbf{Z}, 2 n+2) \\
& p_{1} \downarrow \\
& X \quad \stackrel{k^{3}}{\rightarrow} \quad K(\mathbf{Z}, 3)
\end{aligned}
$$

where the $k$-invariants $k^{3}$ and $k^{2 n+2}$ are given in

LEMMA 2.1. $k^{3}=0, E_{1}=X \times K(\mathbf{Z}, 2)$ and

$$
k^{2 n+2}=\sum_{i=0}^{n+1}(-1)^{i} c_{i}(\xi) \otimes a^{n+1-i}
$$

where $a$ is a generator of $H^{2}(\mathbf{Z}, 2 ; \mathbf{Z})$ and $c_{i}(\xi) \in H^{2 i}(X ; \mathbf{Z}), 1 \leq i \leq n+1$, are the Chern classes of $\xi$.

Proof. Choose an imbedding $i: V \rightarrow X \times \mathbf{C}^{\infty}$ of $\xi$ into the trivial infinite dimensional vector bundle over $X$. The induced map $P(i): P(V)$ $\rightarrow P\left(X \times C^{\infty}\right)=X \times P^{\infty}$ is then an imbedding of $P \xi$ into the trivial infinite-dimensional projective bundle over $X$ and $P(i)^{*}(\lambda)=\lambda_{\xi}$, where $\lambda_{\xi}$ and $\lambda$ are the canonical line bundles ([3], p. 233) over $P(V)$ and $X \times P^{\infty}$ respectively.

Since the restriction of $P(i)$ to the fiber is the usual imbedding of $P^{n}$ into $P^{\infty}$, we may take $E_{1}=X \times P^{\infty}$ and $p_{1}=\mathrm{pr}_{1}: E_{1}=X \times P^{\infty} \rightarrow X$ as the first stage in the Moore-Postnikov factorization of $P \xi$.

By the defining relation ([3], Definition 2.6, p. 234) for the Chern classes of $\xi$, we have

$$
P(i) *\left(\sum_{i=0}^{n+1}(-1)^{i} c_{i}(\xi) \otimes a^{n+1-i}\right)=\sum_{i=0}^{n+1}(-1)^{i} c_{\imath}(\xi) c_{1}\left(\lambda_{\xi}\right)^{n+1-i}=0
$$

for $P(i)^{*}$ is an $H^{*}(X)$-module homomorphism and $P(i)^{*}(1 \otimes a)=$ $P(i)^{*} c_{1}(\lambda)=c_{1}\left(\lambda_{\xi}\right)$. Since $H^{*}(P(V))$ is free $H^{*}(X)$-module by the 
Leray-Hirsch Theorem ([3], Theorem 1.1, p. 231), it follows in fact that

$$
k^{2 n+2}=\sum_{i=0}^{n+1}(-1)^{i} c_{i}(\xi) \otimes a^{n+1-i}
$$

generates $H^{2 n+2}(X \times K(\mathbf{Z}, 2) ; \mathbf{Z}) \cap \operatorname{kern} P(i)^{*}$.

Assume for the rest of this section that $\operatorname{dim} X<2 n+1$. For $i=$ $1,2,3$, let $\Gamma_{i}$ be the space of sections of $E_{i} \rightarrow X$ vertically homotopic to $u_{i}$, where $u_{3}=q u, u_{2}=p_{3} u_{3}$ and $u_{1}=p_{2} u_{2}$. According to $([1], \S 2)$ there is then an induced tower of fibrations

$$
\begin{array}{ccccc} 
& & \Gamma_{u} & & \\
& & & \\
K(\mathbf{Z} / 2,2 n+2)^{X} & \rightarrow & \Gamma_{3} & & \\
& & p_{3} \downarrow & & \\
K(\mathbf{Z}, 2 n+1)^{X} & \rightarrow & \Gamma_{2} & \stackrel{k^{2 n+3}}{\rightarrow} & K(\mathbf{Z} / 2,2 n+3)^{X} \\
& & p_{2} \downarrow & & \\
& & \Gamma_{1} & \underline{k}^{2 n+2} & K
\end{array}
$$

where $\underline{k}^{2 n+i}$ denotes the map defined by composition with $k^{2 n+i}, i=2,3$. Moreover, $p_{3}: \Gamma_{3} \rightarrow \Gamma_{2}$ is the pull-back along $\underline{k}^{2 n+3}$ of the path space fibration over $K(\mathbf{Z} / 2,2 n+3)^{X}$ and $p_{2}: \Gamma_{2} \rightarrow \Gamma_{1}$ is the pull-back along $\underline{k}^{2 n+2}$ of the path space fibration over $K(\mathbf{Z}, 2 n+2)^{X}$.

There is a homotopy equivalence

$$
h: K(\mathbf{Z}, 2) \times F_{0}(X, K(\mathbf{Z}, 2)) \rightarrow \Gamma_{1}
$$

where $F_{0}(X, K(\mathbf{Z}, 2)) \subset K(\mathbf{Z}, 2)^{X}$ denotes the space of based, null-homotopic maps of $X$ into $K(\mathbf{Z}, 2)$. Note that $F_{0}(X, K(\mathbf{Z}, 2))=K\left(H^{1}(X ; \mathbf{Z}), 1\right)$; see e.g. ([1], §1). For $y \in K(\mathbf{Z}, 2), \alpha \in F_{0}(X, K(\mathbf{Z}, 2))$ and $x \in X$, the homotopy equivalence $h$ is given by $h(y, \alpha)(x)=(x, y \cdot \alpha(x) \cdot \mu(x))$, where the multiplication refers to the $H$-space structure of $K(\mathbf{Z}, 2)$ and where $\mu: X \rightarrow K(\mathbf{Z}, 2)$ is the second component of the section $u_{1}: X \rightarrow E_{1}$ $=X \times K(\mathbf{Z}, 2)$.

Via $h$, the adjoint of $\underline{k}^{2 n+2}$ may be identified with a map

$$
f^{2 n+2}: K(\mathbf{Z}, 2) \times K\left(H^{1}(X ; \mathbf{Z}), 1\right) \times X \rightarrow K(\mathbf{Z}, 2 n+2) .
$$

In order to identify $f^{2 n+2}$ as a cohomology class, let $c_{1}(u) \in$ $H^{2}(X ; \mathbf{Z})$ denote $c_{1}\left(u^{*}\left(\lambda_{\xi}\right)\right)=\mu^{*}(a)$, let $\left\{x_{j}\right\}$ be a free basis of $H^{1}(X ; \mathbf{Z})$, and let $\left\{x_{j}^{\prime}\right\}$ be the dual basis of $H^{1}\left(H^{1}(X ; \mathbf{Z}), 1 ; \mathbf{Z}\right)=$ $\operatorname{Hom}\left(H^{1}(X ; \mathbf{Z}), \mathbf{Z}\right)$. 
LEMMA 2.2. The homotopy class of $f^{2 n+2}$ is given by

$$
\begin{aligned}
f^{2 n+2}= & \sum_{i=0}^{n+1}(-1)^{i}\left(1 \otimes 1 \otimes c_{i}(\xi)\right) \\
& \cup\left(a \otimes 1 \otimes 1+1 \otimes 1 \otimes c_{1}(u)+\sum_{j} 1 \otimes x_{j}^{\prime} \otimes x_{j}\right)^{n+1-i} .
\end{aligned}
$$

Proof. Let $g: K(\mathbf{Z}, 2) \times F_{0}(X, K(\mathbf{Z}, 2)) \times X \rightarrow X \times K(\mathbf{Z}, 2)$ be the map given by $g(y, \alpha, x)=(x, y \cdot \alpha(x) \cdot \mu(x))$. Then

$$
\begin{gathered}
g^{*}\left(c_{i}(\xi) \otimes 1\right)=1 \otimes 1 \otimes c_{i}(\xi), \\
g^{*}((1 \otimes a))=a \otimes 1 \otimes 1+1 \otimes 1 \otimes c_{1}(u)+\sum_{j} 1 \otimes x_{j}^{\prime} \otimes x_{j}
\end{gathered}
$$

for it follows from $([\mathbf{1}], \S 1)$ that $e=\sum_{j} x_{j}^{\prime} \otimes x_{j}$, where $e: F_{0}(X, K(\mathbf{Z}, 2)) \times$ $X \rightarrow K(\mathbf{Z}, 2)$ is the evaluation map $e(\alpha, x)=\alpha(x)$.

As $f^{2 n+2}=g^{*}\left(k^{2 n+2}\right)$, Lemma 2.2 is now a consequence of Lemma 2.1.

As a special case of the above result we emphasize

Corollary 2.3. Suppose $H^{1}(X ; \mathbf{Z})=0$. Then

$$
f^{2 n+2}=\sum_{i=0}^{n+1} \sum_{j=0}^{n+1-i}(-1)^{i}\left(\begin{array}{c}
n+1-i \\
j
\end{array}\right) a^{n+1-i-j} \otimes c_{i}(\xi) c_{1}(u)^{j} \text {. }
$$

Let $\Gamma$ denote the space of all sections of $P \xi$. By associating to each section $u \in \Gamma$ the cohomology class $c_{1}(u)=c_{1}\left(u^{*}\left(\lambda_{\xi}\right)\right)$ of the induced line bundle over $X$ we get a map $c_{1}: \pi_{0}(\Gamma) \rightarrow H^{2}(X ; \mathbf{Z})$ from the set $\pi_{0}(\Gamma)$ of (path-)components of $\Gamma$ to $H^{2}(X ; \mathbf{Z})$. Since $c_{1}(u)=\mu^{*}(a)$, an easy application of obstruction theory shows

Proposition 2.4. The map

$$
c_{1}: \pi_{0}(\Gamma) \rightarrow H^{2}(X ; \mathbf{Z})
$$

is bijective when $\operatorname{dim} X<2 n+1$.

With this classification of the set of vertical homotopy classes of sections of $P \xi$, we conclude $\S 2$. The following sections contain examples of applications of the above results to the computation of the homology of $\Gamma_{u}$. 
3. Sections of projective bundles over surfaces. Suppose $X=T$ is a closed, orientable surface of genus $g \geq 0$. By Proposition 2.4, the space $\Gamma$ of sections of the projective bundle $P \xi: P(V) \rightarrow T$ has a countably infinite number of components classified by $H^{2}(T ; \mathbf{Z})$. The component $\Gamma_{u} \subset \Gamma$ containing the section $u: T \rightarrow P(V)$ determines as in $\$ 2$ a sequence of fibrations

$$
\begin{aligned}
& \Gamma_{u} \\
& q \downarrow \\
& \prod_{i=0}^{2} K\left(H^{2-i}(T ; \mathbf{Z} / 2), 2 n+i\right) \quad \rightarrow \Gamma_{3} \\
& p_{3} \downarrow \\
& \prod_{i=0}^{2} K\left(H^{2-i}(T ; \mathbf{Z}), 2 n+i-1\right) \quad \rightarrow \Gamma_{2} \\
& p_{2} \downarrow \\
& K(\mathbf{Z}, 2) \times K\left(H^{1}(T ; \mathbf{Z}), 1\right) \quad=\Gamma_{1} \stackrel{\underline{k}^{2 n+2}}{\rightarrow} \quad \prod_{i=0}^{2} K\left(H^{2-i}(T ; \mathbf{Z}), 2 n+i\right)
\end{aligned}
$$

where we have identified the fibers as well as the space $K(\mathbf{Z}, 2 n+2)^{T}$ with products of Eilenberg-MacLane spaces $([1], \S 1)$. For $i=0,1,2$, let

$$
\underline{k}_{i}^{2 n+2} \in H^{2 n+i}\left(\Gamma_{1} ; \mathbf{Z}\right) \otimes H^{2-i}(T ; \mathbf{Z})
$$

be the components of $\underline{k}^{2 n+2}$ corresponding to the splitting of

$$
K(\mathbf{Z}, 2 n+2)^{T} \text {. }
$$

Choose generators $A_{j}, B_{j} \in H^{1}(T ; \mathbf{Z}), 1 \leq j \leq g$, such that $A_{i} A_{j}=$ $B_{i} B_{j}=0$ and $A_{i} B_{j}=\delta_{i j} U$, where $U$ generates $H^{2}(T ; \mathbf{Z})$, and let as before $A_{j}^{\prime}, B_{j}^{\prime}$ be the dual generators of $H^{1}\left(H^{1}(T ; \mathbf{Z}), 1 ; \mathbf{Z}\right)$.

LEMMA 3.1. The components $\underline{k}_{i}^{2 n+2}$ of $\underline{k}^{2 n+2}$ are

$$
\begin{aligned}
\underline{k}_{0}^{2 n+2}= & (n+1) a^{n} \otimes 1 \otimes c_{1}(u)-a^{n} \otimes 1 \otimes c_{1}(\xi) \\
& -n(n+1) a^{n-1} \otimes \sum_{j=1}^{g} A_{j}^{\prime} B_{j}^{\prime} \otimes U \\
\underline{k}_{1}^{2 n+2}= & (n+1) \sum_{j=1}^{g}\left(a^{n} \otimes A_{j}^{\prime} \otimes A_{j}+a^{n} \otimes B_{j}^{\prime} \otimes B_{j}\right) \\
\underline{k}_{2}^{2 n+2}= & a^{n+1} \otimes 1 \otimes 1 .
\end{aligned}
$$

Proof. Let

$$
e=\sum_{j=1}^{g}\left(A_{j}^{\prime} \otimes A_{j}+B_{j}^{\prime} \otimes B_{j}\right) \in H^{2}\left(F_{0}(T, K(\mathbf{Z}, 2)) \times T ; \mathbf{Z}\right)
$$


be the evaluation map $e: F_{0}(T, K(\mathbf{Z}, 2)) \times T \rightarrow K(\mathbf{Z}, 2)$. Then

$$
e^{2}=-2 \sum_{j=1}^{g} A_{j}^{\prime} B_{j}^{\prime} \otimes U
$$

while $e^{3}=0$. Lemma 2.2 then shows that the adjoint $f^{2 n+2}$ of $\underline{k}^{2 n+2}$ is

$$
\begin{aligned}
f^{2 n+2}= & \left(a \otimes 1 \otimes 1+1 \otimes 1 \otimes c_{1}(u)+1 \otimes e\right)^{n+1} \\
& -\left(1 \otimes 1 \otimes c_{1}(\xi)\right)\left(a \otimes 1 \otimes 1+1 \otimes 1 \otimes c_{1}(u)+1 \otimes e\right)^{n} \\
= & a^{n+1} \otimes 1 \otimes 1+(n+1) a^{n} \otimes 1 \otimes c_{1}(u)-a^{n} \otimes 1 \otimes c_{1}(\xi) \\
& +(n+1) a^{n} \otimes \sum_{j=1}^{g}\left(A_{j}^{\prime} \otimes A_{j}+B_{j}^{\prime} \otimes B_{j}\right) \\
& -n(n+1) a^{n-1} \otimes \sum_{j=1}^{g} A_{j}^{\prime} B_{j}^{\prime} \otimes U
\end{aligned}
$$

and from this formula we can read off $([1], \S 1)$ the expressions for $\underline{k}_{i}^{2 n+2}$, $i=0,1,2$.

Let $t(u) \in \mathbf{Z}$ be the integer determined by the equation

$$
t(u) U=(n+1) c_{1}(u)-c_{1}(\xi)
$$

in $H^{2}(T ; \mathbf{Z})$. Then we may write

$$
\underline{k}_{0}^{2 n+2}=t(u) a^{n} \otimes 1 \otimes U-n(n+1) a^{n-1} \otimes \sum_{j=1}^{g} A_{j}^{\prime} B_{j}^{\prime} \otimes U .
$$

As the main result of this section now follows

THEOREM 3.2. The first $2 n-1$ integral homology groups of $\Gamma_{u}$ are given by

$$
H_{r}\left(\Gamma_{u} ; \mathbf{Z}\right)= \begin{cases}H_{r}\left(\Gamma_{1} ; \mathbf{Z}\right), & 0 \leq r<2 n-1, \\ H_{2 n-1}\left(\Gamma_{1} ; \mathbf{Z}\right) \oplus Z_{u}, & r=2 n-1,\end{cases}
$$

where $Z_{u}=\mathbf{Z} /|t(u)| \otimes \mathbf{Z} / n(n+1)$ if $g>0$ and $Z_{u}=\mathbf{Z} /|t(u)|$ if $T=S^{2}$ is the 2-sphere.

Proof. Since the fibres of both $q: \Gamma_{u} \rightarrow \Gamma_{3}$ and $p_{3}: \Gamma_{3} \rightarrow \Gamma_{2}$ have vanishing reduced integral cohomology groups in dimension $\leq 2 n$, it follows that $H^{r}\left(\Gamma_{u} ; \mathbf{Z}\right)=H^{r}\left(\Gamma_{2} ; \mathbf{Z}\right)$ for $r \leq 2 n$.

To compute $H^{r}\left(\Gamma_{2} ; \mathbf{Z}\right)$ we consider the Leray-Serre cohomology 
spectral sequence $\left\{E_{s}^{p q}\right\}$ with integral coefficients associated to $p_{2}: \Gamma_{2} \rightarrow$ $\Gamma_{1}$. We have $E_{2}^{p q}=0$ for $0<q<2 n-1$ while $E_{2}^{0,2 n-1} \cong \mathbf{Z}$ and $E_{2}^{1,2 n-1}$ $\cong H^{1}(T ; \mathbf{Z}) \cong E_{2}^{0,2 n}$. Since $p_{2}: \Gamma_{2} \rightarrow \Gamma_{1}$ is induced by $\underline{k}^{2 n+2}$ from the path space fibration over $K(\mathbf{Z}, 2 n+2)^{T}$, the first non-trivial differential $d_{2 n}$ : $E_{2}^{0,2 n-1} \rightarrow E_{2}^{2 n, 0}$ is determined by $\underline{k}_{0}^{2 n+2}$. Assuming that $g>0$, we conclude that $E_{\infty}^{0,2 n-1}=0$ while

$$
E_{\infty}^{2 n, 0}=H^{2 n}\left(\Gamma_{1} ; \mathbf{Z}\right) / \mathbf{Z} \cdot\left(t(u) a^{n} \otimes 1-n(n+1) a^{n-1} \otimes \sum A_{j}^{\prime} B_{j}^{\prime}\right) .
$$

It follows that $H^{r}\left(\Gamma_{u} ; \mathbf{Z}\right)=H^{r}\left(\Gamma_{1} ; \mathbf{Z}\right)$ for $0 \leq r \leq 2 n-1$. Moreover, since $E_{2}^{1,2 n-1}$ and $E_{2}^{0,2 n}$ are free abelian groups, the torsion subgroup of $H^{2 n}\left(\Gamma_{u} ; \mathbf{Z}\right)$ equals the torsion subgroup, $\mathbf{Z} /|t(u)| \otimes \mathbf{Z} / n(n+1)$, of $E_{\infty}^{2 n, 0}$.

Using the extra information contained in Lemma 3.1, the reader may carry the analysis of the spectral sequence a little further and compute $H^{2 n}\left(\Gamma_{u} ; \mathbf{Z}\right)$.

Let $R_{u}$ be the subring of $H^{*}\left(\Gamma_{u} ; \mathbf{Z}\right)$ generated by all cohomology classes of degree $\leq 2$ and let $T_{u}$ be $R_{u}$ truncated above degree $2 n$. The proof of Theorem 3.2 shows that

$$
T_{u}=\mathbf{Z}[a] \otimes \Lambda\left(A_{1}^{\prime}, B_{1}^{\prime}, \ldots, A_{g}^{\prime}, B_{g}^{\prime}\right) / I_{u}
$$

where $I_{u} \subset \mathbf{Z}[a] \otimes \Lambda\left(A_{1}^{\prime}, B_{1}^{\prime}, \ldots, A_{g}^{\prime}, B_{g}^{\prime}\right)$ is the ideal generated by

$$
t(u) a^{n} \otimes 1-n(n+1) a^{n-1} \otimes \sum A_{j}^{\prime} B_{j}^{\prime}
$$

together with all elements of degree $>2 n . T_{u}$ is a homotopy invariant of $\Gamma_{u}$, so from the fact (pointed out to me by A. Thorup) that

$$
T_{u}^{2 n} /\left\langle T_{u}^{1}\right\rangle^{2 n}=\mathbf{Z} /|t(u)|,
$$

where $\left\langle T_{u}^{1}\right\rangle$ is the ideal of $T_{u}$ generated by all elements of degree 1 , we obtain

Corollary 3.3. Let $v: T \rightarrow P(V)$ be a section that is not vertically homotopic to $u$. If $\Gamma_{u}$ is homotopy equivalent to $\Gamma_{v}$, then

$$
(n+1)\left(c_{1}(u)+c_{1}(v)\right)=2 c_{1}(\xi) .
$$

As a special case we take as $\xi$ the trivial $(n+1)$-dimensional vector bundle over $T$. Then $\Gamma=M\left(T, P^{n}\right)$ is the space of maps of $T$ into $P^{n}$ and $\Gamma_{u}=M_{k}\left(T, P^{n}\right)$ is the component consisting of maps of degree $k=c_{1}(u)$.

Corollary 3.4. Two components of $M\left(T, P^{n}\right)$ are homotopy equivalent if and only if their associated degrees have the same absolute value. 
This result was also obtained for $n=1$ in [2] and for $g=0$ in [5]. In the nonorientable case we get

Proposition 3.5. Suppose that the base space $X=U_{h}$ is a closed, nonorientable surface of genus $h>1$. Then there is a $(2 n-1)$-connected map

$$
p_{1}: \Gamma_{u} \rightarrow \Gamma_{1}=K(\mathbf{Z}, 2) \times K\left(\mathbf{Z}^{h-1}, 1\right)
$$

and

$$
H_{2 n-1}\left(\Gamma_{u} ; \mathbf{Z} / 2\right) \cong H_{2 n-1}\left(\Gamma_{1} ; \mathbf{Z} / 2\right) \oplus Z_{u}
$$

where

$$
Z_{u}= \begin{cases}\mathbf{Z} / 2 & \text { if }(n+1) c_{1}(u)=c_{1}(\xi) \\ 0 & \text { if }(n+1) c_{1}(u) \neq c_{1}(\xi)\end{cases}
$$

In particular the two components of $\Gamma_{u}$ are not homotopy equivalent when $n$ is even.

Depending on knowledge of the cup square $e^{2}$, the above method actually makes possible the computation of the first $2 n-1$ homology groups of $\Gamma_{u}$ when the base space $X$ is any 2-dimensional CW-complex.

4. Sections of projective bundles over projective spaces. In this section we assume that the base space $X=P^{m}$ is the complex projective $m$-space, $1 \leq m \leq n$. The space of sections $\Gamma$ then has a countably infinite number of components classified by $H^{2}\left(P^{m} ; \mathbf{Z}\right)$. The component $\Gamma_{u} \subset \Gamma$, containing the section $u: P^{m} \rightarrow P(V)$, determines a tower of fibrations

$$
\begin{array}{rlc} 
& \Gamma_{u} & \\
& & \downarrow \\
\prod_{i=0}^{m} K(\mathbf{Z} / 2,2 n-2 m+2+2 i) & \rightarrow & \Gamma_{3} \\
& & \downarrow \\
\prod_{i=0}^{m} K(\mathbf{Z}, 2 n-2 m+1+2 i) & \rightarrow & \Gamma_{2} \\
& & \downarrow \\
K(\mathbf{Z}, 2) & = & \Gamma_{1} \stackrel{\underline{k}^{2 n+2}}{\rightarrow} \prod_{i=0}^{m} K(\mathbf{Z}, 2 n-2 m+2+2 i)
\end{array}
$$

where the fibers are products of Eilenberg-MacLane spaces. 
Let $a_{m} \in H^{2}\left(P^{m} ; \mathbf{Z}\right)$ be a generator and let $t(u) \in \mathbf{Z}$ be the integer determined by the equation

$$
t(u) a_{m}^{m}=\sum_{i=0}^{m}(-1)^{i}\left(\begin{array}{c}
n+1-i \\
n+1-m
\end{array}\right) c_{i}(\xi) c_{1}(u)^{m-i}
$$

in $H^{2 m}\left(P^{m} ; \mathbf{Z}\right)$. Then by Corollary 2.3 , the first component $\underline{k}_{0}^{2 n+2} \in$ $H^{2 n-2 m+2}(\mathbf{Z}, 2 ; \mathbf{Z}) \otimes H^{2 m}\left(P^{m} ; \mathbf{Z}\right)$ of the map $\underline{k}^{2 n+2}$ is

$$
\underline{k}_{0}^{2 n+2}=t(u) a^{n-m+1} \otimes a_{m}^{m} .
$$

By a spectral sequence argument similar to that of $\S 3$ we get

THEOREM 4.1. For $0 \leq r<2 n-2 m+1, H^{r}\left(\Gamma_{u} ; \mathbf{Z}\right)=H^{r}(\mathbf{Z}, 2 ; \mathbf{Z})$, while the $(2 n-2 m+1)$ - and $(2 n-2 m+2)$-dimensional integral cohomology groups of $\Gamma_{u}$ are determined by the exact sequence

$$
0 \rightarrow H^{2 n-2 m+1}\left(\Gamma_{u}\right) \rightarrow \mathbf{Z} \stackrel{d_{u}}{\rightarrow} \mathbf{Z} \rightarrow H^{2 n-2 m+2}\left(\Gamma_{u}\right) \rightarrow 0
$$

where $d_{u}$ is multiplication by $t(u)$.

With the trivial $(n+1)$-plane bundle as $\xi$, this yields

COROllary 4.2. For $k \in \mathbf{Z}$, let $M_{k}\left(P^{m}, P^{n}\right)$ be the space of maps of degree $k$ of $P^{m}$ into $P^{n}, 1 \leq m \leq n$. Then

$$
H_{2 n-2 m+1}\left(M_{k}\left(P^{m}, P^{n}\right) ; \mathbf{Z}\right)=\mathbf{Z} /\left(\begin{array}{c}
n+1 \\
m
\end{array}\right)|k|^{m} \text {. }
$$

This result, which also was obtained in [5] by a different method, shows that two components of $M\left(P^{m}, P^{n}\right)$ are homotopy equivalent if and only if their associated degrees have the same absolute value.

5. Sections of projective bundles over lens spaces. As an example where the space $\Gamma$ has a finite number of components we shall here consider $X=L^{2 m+1}(p)$, the lens space obtained by letting $\mathbf{Z} / p$ act on $S^{2 m+1}$ in the usual way. Throughout this section we assume that $1 \leq m<n$ and that $p$ is odd. By Proposition 2.4, the components of $\Gamma$ are classified by $H^{2}\left(L^{2 m+1}(p) ; \mathbf{Z}\right) \cong \mathbf{Z} / p$.

As above, let $\Gamma_{u} \subset \Gamma$ be the component containing the section $u$ : $L^{2 m+1}(p) \rightarrow P(V)$ of $P \xi$. After inserting an extra stage in the MoorePostnikov decomposition of $P \xi$ and noting that

$$
H^{r}\left(L^{2 m+1}(p) ; \mathbf{Z} / 2\right)= \begin{cases}\mathbf{Z} / 2, & r=0,2 m+1, \\ 0, & \text { otherwise }\end{cases}
$$


we obtain the following tower of fibrations

$$
\begin{array}{rll} 
& & \Gamma_{u} \\
K(\mathbf{Z} / 2, t+2) \times K(\mathbf{Z} / 2,2 n+3) & \rightarrow & \Gamma_{4} \\
K(\mathbf{Z} / 2, t+1) \times K(\mathbf{Z} / 2,2 n+2) & \rightarrow & \Gamma_{3} \\
K(\mathbf{Z}, t) \times \prod_{i=0}^{m} K(\mathbf{Z} / p, t+1+2 i) & \rightarrow & \Gamma_{2} \\
& & \downarrow \\
K(\mathbf{Z}, 2) & =\Gamma_{1} \stackrel{\underline{k}^{2 n+2}}{\rightarrow} K(\mathbf{Z}, t+1) \times \prod_{i=0}^{m} K(\mathbf{Z} / p, t+2+2 i)
\end{array}
$$

where $t=2 n-2 m$. Since

$$
H^{r}(K(\mathbf{Z} / 2, t+1) \times K(\mathbf{Z} / 2,2 n+1+i) ; \mathbf{Z} / p)=0
$$

for $0 \leq r \leq t+2, i=1,2$, this implies

LemMa 5.1. $H^{r}\left(\Gamma_{u} ; \mathbf{Z} / p\right)=H^{r}\left(\Gamma_{2} ; \mathbf{Z} / p\right)$ for $0 \leq r \leq 2 n-2 m+2$.

Let $a_{m} \in H^{2}\left(L^{2 m+1}(p) ; \mathbf{Z}\right)$ be a generator and choose $t(u) \in \mathbf{Z}$ such that $0 \leq t(u)<p$ and

$$
t(u) a_{m}^{m}=\sum_{i=0}^{m}(-1)^{i}\left(\begin{array}{c}
n+1-i \\
n+1-m
\end{array}\right) c_{i}(\xi) c_{1}(u)^{m-i}
$$

in $H^{2 m}\left(L^{2 m+1}(p) ; \mathbf{Z}\right) \cong \mathbf{Z} / p$. The first non-trivial component

$$
k_{0}^{2 n+2}: \Gamma_{1} \rightarrow K\left(H^{2 m}\left(L^{2 m+1}(p) ; \mathbf{Z}\right), 2 n-2 m+2\right)
$$

of $\underline{k}^{2 n+2}$ is then, by Corollary 2.3 , given by

$$
\underline{k}_{0}^{2 n+2}=t(u) a^{n-m+1} \otimes a_{m}^{m} .
$$

Combining this with Lemma 5.1, we can prove

THeOREM 5.2. When the base space $X=L^{2 m+1}(p), 1 \leq m<n, p$ odd, is a lens space, we have:

(i) $H^{r}\left(\Gamma_{u} ; \mathbf{Z}\right)=H^{r}(\mathbf{Z}, 2 ; \mathbf{Z})$ for $0 \leq r<2 n-2 m$.

(ii) $H^{2 n-2 m}\left(\Gamma_{u} ; \mathbf{Z}\right)=\mathbf{Z} \oplus \mathbf{Z}$.

(iii) $H^{2 n-2 m+1}\left(\Gamma_{u} ; \mathbf{Z} / p\right)=\mathbf{Z} / t(u) \otimes \mathbf{Z} / p$.

(iv) There is a filtration

$$
\mathbf{Z} / t(u) \otimes \mathbf{Z} / p=J^{0} \subset J^{1} \subset J^{2}=H^{2 n-2 m+2}\left(\Gamma_{u} ; \mathbf{Z} / p\right)
$$

where $J^{1} / J^{0} \cong J^{2} / J^{1} \cong \mathbf{Z} / p$. 
Proof. The first two assertions follows easily from the constructed tower of fibrations. To prove the remaining cases we can, according to Lemma 5.1, use the cohomology spectral sequence $\left\{E_{r}^{s t}\right\}$ with $\mathbf{Z} / p$-coefficients associated to $p_{2}: \Gamma_{2} \rightarrow \Gamma_{1}$. Note that $E_{2}^{s t}=0$ when $0<t<2 n-2 m$ or $s$ is odd. The differentials $d_{2}: E_{2}^{0,2 n-2 m+2} \rightarrow E_{2}^{2,2 n-2 m+1}$ and $d_{2}$ : $E_{2}^{0,2 n-2 m+1} \rightarrow E_{2}^{2,2 n-2 m}$ are trivial for so are the corresponding differentials in the spectral sequence for the path space fibration from which $p_{2}$ : $\Gamma_{2} \rightarrow \Gamma_{1}$ is induced. The only non-trivial relevant differential is thus $d_{2 n-2 m+2}: E_{2}^{0,2 n-2 m+1} \rightarrow E_{2}^{2 n-2 m+2,0}$ which is determined by $\underline{k}_{0}^{2 n+2}$; i.e. there is an exact sequence

$$
0 \rightarrow E_{\infty}^{0,2 n-2 m+1} \rightarrow \mathbf{Z} / p \stackrel{d_{u}}{\rightarrow} \mathbf{Z} / p \rightarrow E_{\infty}^{2 n-2 m+2,0} \rightarrow 0
$$

where $d_{u}$ is multiplication by $t(u)$. This proves (iii). To prove (iv), we note that $E_{\infty}^{0,2 n-2 m+2}=E_{2}^{0,2 n-2 m+2}=\mathbf{Z} / p$ and $E_{\infty}^{2,2 n-2 m}=E_{2}^{2,2 n-2 m}=\mathbf{Z} / p$.

For any $k \in \mathbf{Z}$, let $M_{k}\left(L^{2 m+1}(p), P^{n}\right)$ be the space of maps of degree $k \bmod p$ of $L^{2 m+1}(p)$ into $P^{n}$.

Corollary 5.3. If $M_{k}\left(L^{2 m+1}(p), P^{n}\right)$ is homotopy equivalent to $M_{l}\left(L^{2 m+1}(p), P^{n}\right)$, then

$$
\operatorname{gcd}\left(\left(\begin{array}{c}
n+1 \\
m
\end{array}\right)|k|^{m}, p\right)=\operatorname{gcd}\left(\left(\begin{array}{c}
n+1 \\
m
\end{array}\right)|l|^{m}, p\right) .
$$

The above necessary condition only provides a partial solution to the homotopy classification problem for the components of the space $M\left(L^{2 m+1}(p), P^{n}\right)$ of maps of $L^{2 m+1}(p)$ into $P^{n}$. The complete solution is unknown.

After finishing this manuscript I learned that the results stated in Corollary 3.4 and in the remark immediately after Corollary 4.2 also have been obtained by M. C. Crabb and W. A. Sutherland.

\section{REFERENCES}

[1] A. Haefliger, Rational homotopy of the space of sections of a nilpotent bundle, Trans. Amer. Math. Soc., 273 (1982), 609-620.

[2] V. L. Hansen, On the space of maps of a closed surface into the 2-sphere, Math. Scand., 35 (1974), 149-158.

[3] D. Husemoller, Fibre Bundles, Second Edition. Graduate Texts in Mathematics 20. Springer-Verlag 1975. 
[4] L. L. Larmore and E. Thomas, On the fundamental group of a space of sections, Math. Scand., 47 (1980), 232-246.

[5] J. M. Møller, On spaces of maps between complex projective spaces, Proc. Amer. Math. Soc., (to appear).

Received May 5, 1983.

UNIVERSITY OF COPENHAGEN

UNIVERSITETSPARKEN 5

DK - 2100 KøBENHAVN $\varnothing$

DENMARK 


\section{PACIFIC JOURNAL OF MATHEMATICS \\ EDITORS}

Donald BABBITT (Managing Editor)

University of California

Los Angeles, CA 90024

J. Dugundu

University of Southern Californa

Los Angeles, CA 90089-1113

R. FINN

Stanford University

Stanford, CA 94305

HermanN FLaSChKa

University of Arizona

Tucson, AZ 85721

\author{
C. C. MOORE \\ University of California \\ Berkeley, CA 94720 \\ ARTHur Ogus \\ University of California \\ Berkeley, CA 94720 \\ Hugo Rossi \\ University of Utah \\ Salt Lake City, UT 84112 \\ H. SAMELSON \\ Stanford University \\ Stanford, CA 94305
}

ASSOCIATE EDITORS
R. ARENS
E. F. BECKENBACH
B. H. NeUmanN
F. WOLF
K. YOSHIDA (1906-1982)

\section{SUPPORTING INSTITUTIONS}

UNIVERSITY OF ARIZONA

UNIVERSITY OF BRITISH COLUMBIA

UNIVERSITY OF OREGON

CALIFORNIA INSTITUTE OF TECHNOLOGY

UNIVERSITY OF CALIFORNIA

MONTANA STATE UNIVERSITY

UNIVERSITY OF SOUTHERN CALIFORNIA

UNIVERSITY OF NEVADA, RENO

STANFORD UNIVERSITY

UNIVERSITY OF HAWAII

NEW MEXICO STATE UNIVERSITY

UNIVERSITY OF TOKYO

UNIVERSITY OF UTAH

WASHINGTON STATE UNIVERSITY

OREGON STATE UNIVERSITY

UNIVERSITY OF WASHINGTON 


\section{Pacific Journal of Mathematics}

\section{Vol. 116, No. $1 \quad$ November, 1985}

K. Adachi, Le problème de Lévi pour les fibrés grassmanniens et les variétés

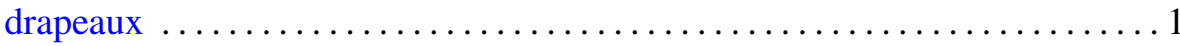

John MacLeod Ball, Remarks on the paper: "Basic calculus of variations" . . . 7 John Kelly Beem and Phillip E. Parker, Whitney stability of solvability . . . 11 Alberto Facchini, Decompositions of algebraically compact modules .......25

S. S. Khare, Finite group action and equivariant bordism $\ldots \ldots \ldots \ldots . \ldots 39$

Horst Leptin, A new kind of eigenfunction expansions on groups $\ldots \ldots \ldots . .45$

Pei-Kee Lin, Unconditional bases and fixed points of nonexpansive

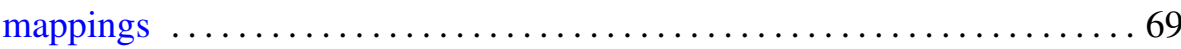

Charles Livingston, Stably irreducible surfaces in $S^{4} \ldots \ldots \ldots \ldots \ldots 77$

Kevin Mor McCrimmon, Nonassociative algebras with scalar involution . . .885

Albert Milani, Singular limits of quasilinear hyperbolic systems in a

bounded domain of $\mathbf{R}^{3}$ with applications to Maxwell's equations

Takemi Mizokami, On $M$-structures and strongly regularly stratifiable

spaces

Jesper M. Møller, On the homology of spaces of sections of complex

projective bundles

Nikolaos S. Papageorgiou, Carathéodory convex integrand operators and

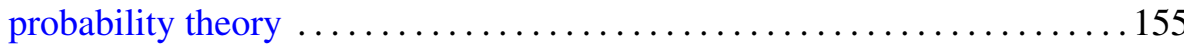

Robert John Piacenza, Transfer in generalized prestack cohomology 185

Lance W. Small and Adrian R. Wadsworth, Integrality of subrings of matrix rings ...

James Michael Wilson, On the atomic decomposition for Hardy spaces 